\title{
Significance of the Solidifying Zone on the Ingot Solidification*
}

\author{
By Tadayoshi TAKAHASHI, ${ }^{* *}$ Iwao HAGIWARA, ${ }^{* * *}$ and Kiyoshi ICHIKAWA.**
}

\section{Synopsis}

The solidifying zone, which is composed of solid and liquid phases at the solidification front, is very important to understand the solidification mechanism of the ingot. This zone has therefore been examined on the basis of the behaviour of the solute-rich liquid between dendrites. The fundamental analysis made for the solidifying zone has been applied to explain the solidification phenomena of steel ingots. The results obtained are summarized as follows:

The effective distribution coefficient, $k^{*}$, is represented by

$$
k^{*}=1-E U
$$

where $E$ is an experimental constant and $U$ is the flow velocity of bulk liquid. Thus $k^{*}$ depends only on $U$. However, there is a minimum in the value of $k^{*}$. The minimum effective distribution coefficient, $k_{m}^{*}$, is expressed by

$$
k_{m}^{*}=1-S p\left(1-k_{o}\right)
$$

where Sp is the critical fraction of solid above which the liquid is completely entrapped by the solid and $k_{o}$ is the equilibrium distribution coefficient. Since the value of $k_{m}^{*}$ is 0.45 for $\mathrm{Al}-4 \mathrm{wt} \% \mathrm{Cu}$ alloy, the value of $S p$ is calculated to be $67 \%$. This agrees well with the value of Sp actually observed for carbon steel, 65 to $69 \%$. Assuming that the value of $S p$ is equal to 0.67, the minimum effective distribution coefficients calculated for the alloying elements in steel are 0.42 for carbon, 0.81 for manganese, 0.37 for phosphorous, and 0.34 for sulphur respectively. These values agree well with the minimum effective distribution coefficients observed for the rim-zone of rimming ingots. From these, it is evident that the macrosegregation in the rim-zone of rimmed steel ingots is determined only by the degree of washing against the solidifying zone with the turbulent flow of bulk liquid.

\section{Introduction}

The dendritic crystals are generally formed during solidification, where the morphology of growing crystals is determined by heat transfer and by crystallographic and physical properties of the alloy. The solidification proceeds with the development of the dendritic aggregates. On the other hand the fluid motion of bulk liquid is caused by the phenomena taken place during the solidification of steel ingot such as free convection, rimming action with the evolution of $\mathrm{CO}$ gas, and so on. Thus the relation between the dendrite morphology and the fluid motion should not be neglected in the discussion of the segregation in steel ingots.

The theoretical equation introduced to the assumption that the solidification interface is planar, can be adopted for the controlled solidification under the conditions of a steep temperature gradient and a constant rate of solidification, ${ }^{1-4)}$ e.g. in the case of zone melting. However, it can not be applied to the solidification of commercial steel ingot, which is naturally solidified in a mold. ${ }^{5-7)}$
A region, in which the dendritic aggregates are in equilibrium with the neighbouring liquid, is formed during the solidification of alloy according to the mutual relation between the temperature profile of the region and the difference in the liquidus and the solidus temperatures. The region is called as "the solidifying zone" in the present paper. In the current investigation the fundamental analysis made for the solidifying zone is also applied to the solidification of steel ingots. A model experiment is firstly performed on the effect of washing against the solidifying zone with the fluid flow of bulk liquid. The segregation behaviour and the solidification mechanism of steel ingots are then discussed on the basis of the results obtained.

\section{Fundamental Concept of the Solidifying Zone}

A region which is composed of the dendrites and the interdendritic liquid is generally formed during the solidification of alloy. This region of partial solidification is called as "the solidifying zone" in this paper. In this chapter the difference between the solidification with the planar interface and that with the solidifying zone is discussed on the basis of the experimental results obtained.

It has been accepted that the transition from the columnar to the equiaxed zone in an ingot can be explained by considering the region of constitutional supercooling, which is determined from the mutual relation between the gradients of temperature and concentration at the solidification front based on the assumption that the solid/liquid interface is planar. In the solidification with a planar interface, the solute concentration of the liquid at the solidification front increases with increasing rate of solidification, and the solute concentration of the solid at the interface is determined from that of the neighbouring solute-rich liquid according to the phase diagram. Thus the distribution of the solute concentration of the solid is smooth so long as the rate of solidification does not change rapidly. Therefore the microsegregation does not occur. It seems that there is no freezing range in the solidification with a planar interface, since the planar interface advances continuously at the liquidus temperature which corresponds with the solute concentration of the liquid.

However, the following four characteristics are seen in the solidification of commercial steel ingots.

(1) There are such freezing ranges as $F_{1}$ and $F_{2}$

* Received November 4, 1971

** Department of Metallurgical Engineering, Faculty of Engineering, Hokkaido University, Sapporo 060.

*** Department of Mechanical Engineering, Hokkaido Institute of Technology, Sapporo 061-24. 


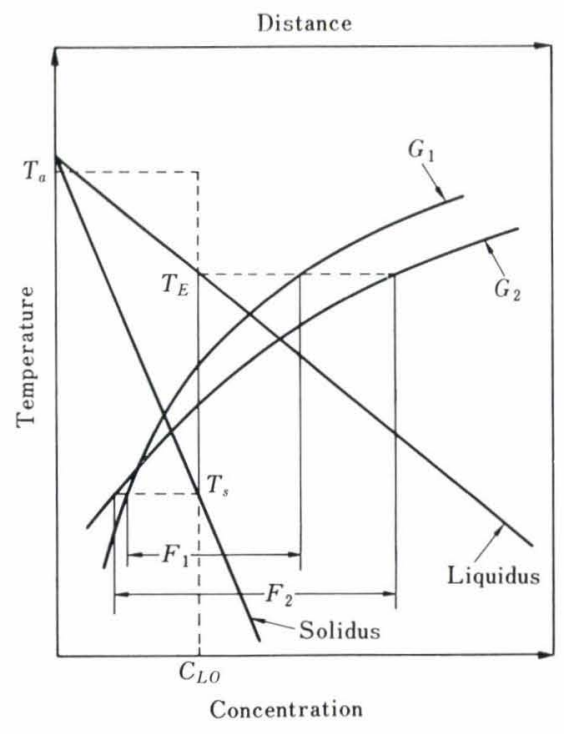

Fig. 1. A schematic illustration of the changes of the temperature profile and the solidifying zone with the progress of solidification.

The subscripts " 1 " and " 2 " show the temperature profile, $G$, and the solidifying zone, $F$, in the initial and later stages of solidification respectively.

shown in Fig. 1 so long as the temperature gradient does not become extremely steep at the solidification interface.

(2) Some difference between the liquidus and the solidus temperatures is observed in the cooling curve for each part of the ingot.

(3) The crystal grows with the formation of dendritic aggregates not only in the chill and the columnar zones but also in the branched columnar and the equiaxed zones. Schürmann and Grostschel ${ }^{8)}$ showed that there is a dendrite structure even in the rimmed steel ingots containing $0.049-0.083 \% \mathrm{C}$, using a special etching method developed by Hultgren and Phragmén. ${ }^{9)}$

(4) Results of the concentration analyses with an electron probe microanalyzer show that the solute concentration changes rapidly in the dendrite arm spacings.

As described above, many contradictions are disclosed between the solidification which proceeds with a planar interface and the solidification of commercial steel ingots. Therefore, the existence of the solidifying zone which is the most important for the solidification of steel ingots may be neglected if the theory for the solidification with a planar interface is applied to the solidification of steel ingots.

The difference in the temperatures between the solid and the liquid phases in the solidifying zone is considered to be very small because of their high heat conductivities. Since the solute concentration of the liquid in the solidifying zone is supposed to be almost in equilibrium with that of the neighbouring solid, the concentration gradient can be estimated from the temperature gradient. The thin solute-rich liquid zone of $10^{-1}$ to $10^{-3} \mathrm{~cm}$ in thickness is formed in the solidification with a planar interface due to a steep temperature gradient at the solid/liquid interface, while the temperature gradient in the solidifying zone is very small because the thickness of the zone is more than $1 \mathrm{~cm}$ at the initial stage of solidification $^{10)}$ and increases as the solidification proceeds. Therefore such constitutional supercooling as in the solidification with a planar interface may be difficult to occur in the solidification with a solidifying zone. This must be taken into account for the discussion on the change in the morphology from the columnar to the free crystals. The concentration distribution and the thickness of the solute-rich liquid zone in the solidification with a planar interface are determined by the degree of fluid motion of the bulk liquid passing through the vicinity of the interface. However, the liquid at the inside of the solidifying zone becomes to show a different solute concentration with the increase of the fraction of solid, since the fluid motion can not be taken place at the position over a limited distance from the tip of the zone. Thus free convection is caused in the solidifying zone owing to the difference in the concentrations between the solute-rich liquid and the neighbouring one. ${ }^{11}$ This behaviour of the solute-rich liquid in the solidifying zone becomes important for the discussion of the inverse $V$ segregation of killed steel ingots. ${ }^{12)}$

\section{Effect of Washing against the Solidifying Zone with the Fluid Motion of Bulk Liquid}

Effect of washing against the solidifying zone by the fluid flow of bulk liquid is important to understand the segregation behaviours during the solidification of steel ingots. Thus the degree of stirring of the remaining liquid during solidification is supposed to characterize whether the steel ingot will be rimmed or killed. Theoretical analysis on the basis of the experimental results obtained by using a special method of solidification has been made in order to clarify the effect of the fluid flow of bulk liquid on the solidifying zone. ${ }^{13,14)}$

\section{Experimental Consideration on the Effective Distribution Coefficient}

\section{Experimental Procedure}

There are some different ways in stirring, for example, the electromagnetic stirring, the stirring by high frequency induction, and so on. But the degree of stirring can not be quantitatively shown by these methods. Therefore, the well-known Taylor's vortex method with two concentric cylinders was used to change the flow velocity of bulk liquid. As the outer cylinder was at rest and the water-cooled inner cylinder was rotating, the solidification proceeded from the inner to the outer side. It was shown that this cooling method is very effective for the study of the segregation behaviour of the ingot, since the solute concentration of the remaining liquid does not increase so much in the later stage of solidification and the gravity segregation does not occur owing to the turbulence and the centrifugal force.

As the sample, $\mathrm{Al}-4 \mathrm{wt} \% \mathrm{Cu}$ alloy was used. A schematic view of the experimental apparatus is shown 
in Fig. 2. The outer cylinder used for melting was a graphite crucible of $12 \mathrm{~cm}$ in I.D., $14 \mathrm{~cm}$ in height, and $1 \mathrm{~cm}$ in thickness. A pipe of $2 \mathrm{~cm}$ in diameter made of copper or steel was used as the inner cylinder. The inner cylinder was cooled by flowing water at the rates of $1 / 6$ and $1 / 18 l /$ sec during solidification. The inner cylinder was rotated at the rates of $90,420,650$, and $750 \mathrm{rpm}$ respectively. The rate of solidification was measured from the direct observation of the thickness of solidified layer on the upper surface of the ingot.

When $\mathrm{Al}-4 \mathrm{wt} \% \mathrm{Cu}$ alloy of about $3 \mathrm{~kg}$ was melted in the crucible the height of the melt became $10 \mathrm{~cm}$. After the molten alloy was sufficiently agitated at $700^{\circ} \mathrm{C}$, the heat supply for the melt was stopped. Then the water-cooled inner cylinder was immersed to $4 \mathrm{~cm}$ below the upper surface of the melt and rotated at each cycle of rotation as soon as possible so that the solidification proceeded from the inner to the outer side.

\section{Experimental Results and Analysis}

In this section it will be discussed what determines the effective distribution coefficient, $k^{*}$, in the solidification which proceeds with the solidifying zone. Burton et al. presented the effective distribution coefficient theoretically as follows: ${ }^{1)}$

$$
k^{*}=\frac{k_{o}}{k_{o}+\left(1-k_{o}\right) \exp (-V \delta / D)}
$$

where $k_{o}$ is the equilibrium distribution coefficient, $D$ the diffusion coefficient of solute, $\delta$ the thickness of the

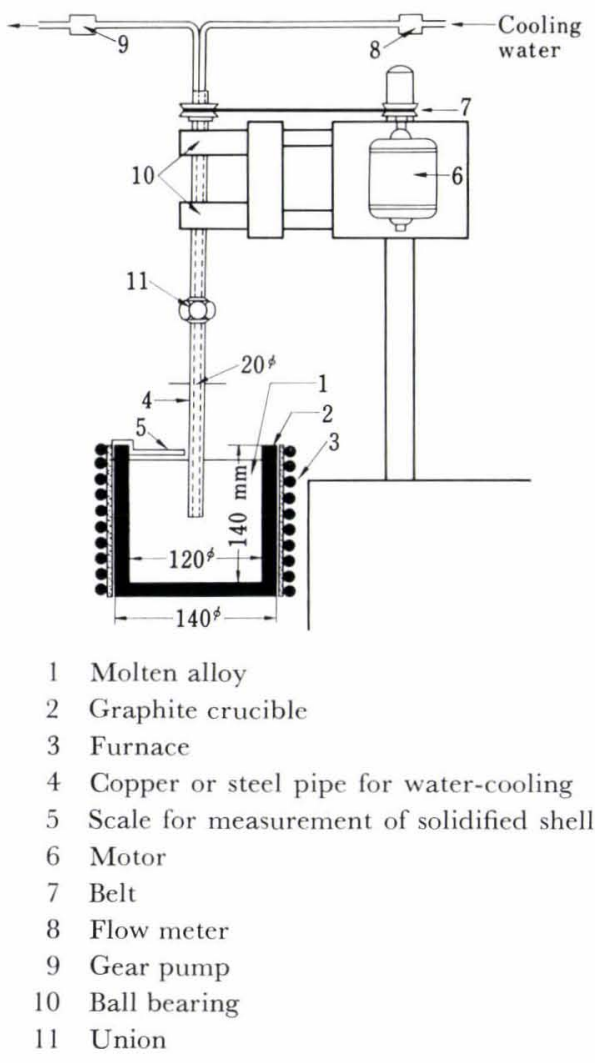

Fig. 2. Experimental apparatus used for the solidification with rotation of the liquid diffusion layer at the planar interface, and $V$ the rate of solidification. In Eq. (1), the factors controlling $k^{*}$ are $\delta$ and $V$ when $k_{o}$ is constant.

The thickness of the laminar sub-layer defined on the basis of hydrodynamics is described as $\delta_{l}$, in order to distinguish $\delta$ from $\delta_{l}$. Now let us examine the mutual relation between $\delta$ and $\delta_{l}$. Substituting $k_{o}=$ $0.16,{ }^{15)} \quad D=7 \times 10^{-5} \mathrm{~cm}^{2} / \mathrm{sec}^{16)}$ and the observed values of $k^{*}$ and $V$ into Eq. (1), the value of $\delta$ at each part of the ingot can be calculated. As the result, the value of $\delta$ remained approximately constant in all the parts of the ingot and the mean value of $\delta$ is $6.0 \times$ $10^{-3} \mathrm{~cm}$.

According to Nikuradse, ${ }^{17}{ }^{7} \delta_{l}$ is expressed as follows:

$$
\delta_{l}=5 \nu \sqrt{ } \rho^{\prime} / \tau
$$

where $\nu$ is the kinematic viscosity, $\rho^{\prime}$ the density, and $\tau$ the shearing stress. Taylor made the experiment on the velocity of the fluid flow between two concentric cylinders and found that the value of $U \times r$ was half the value of $U s \times r_{o}$, where $U$ was the flow velocity, $r$ the distance from the center of the inner cylinder to the fluid, Us the peripheral velocity of the inner cylinder, and $r_{o}$ the radius of the inner cylinder. ${ }^{18)}$ Therefore, the shearing stress is given by

$$
\tau=\frac{\mu U s}{2 \delta_{l}}
$$

where $\mu$ is the viscosity. Using Eqs. (2) and (3), $o_{l}$ is rewritten as follows:

$$
\delta_{l}=50 \nu / U s
$$

The value of $\nu$ is $2.3 \times 10^{-3} \mathrm{~cm}^{2} / \mathrm{sec},{ }^{19)}$ and the value of $U_{s}$ is estimated from both the thickness of the solidified ingot and the cycle of rotation per minute of the inner cylinder during solidification. Substituting these values into Eq. (4), the thickness of the laminar sub-layer is obtained.

The statistical examination ${ }^{13)}$ shows no correlation between the thickness of the diffusion layer, $\delta$, and the thickness of the laminar sub-layer, $\delta_{l}$. The value of $\delta$ is much greater than $\delta_{l}, e . g$. the mean value of $\delta$ is five times larger than that of $\delta_{l}$ at $U s=100 \mathrm{~cm} / \mathrm{sec}$.

If $\delta$ is independent of the degree of stirring, $U s$, the effective distribution coefficient of Eq. (1) should be expressed as a function of the rate of solidification. Figure 3 shows the relation between the effective distribution coefficient, $k^{*}$, the rate of solidification, $V$, and the peripheral velocity of the rotating solid surface, $U s$, for $\mathrm{Al}-4 \mathrm{wt} \% \mathrm{Cu}$ alloy. It might be considered as if $k^{*}$ were related to $V$. However, the detailed examination clearly shows that $k^{*}$ depends only on $U s$ as shown in Fig. 4. The value of $k^{*}$ decreases linearly with increasing $U s$ when $U s$ is decreased below $160 \mathrm{~cm} / \mathrm{sec}$, but remains constant at 0.45 when $U_{S}$ is increased over $160 \mathrm{~cm} / \mathrm{sec}$.

As described above in the solidification with the fluid motion of bulk liquid $k^{*}$ is independent of $V$ but is a function of $U s$ or $U$ only. The empirical equation is expressed as follows: 


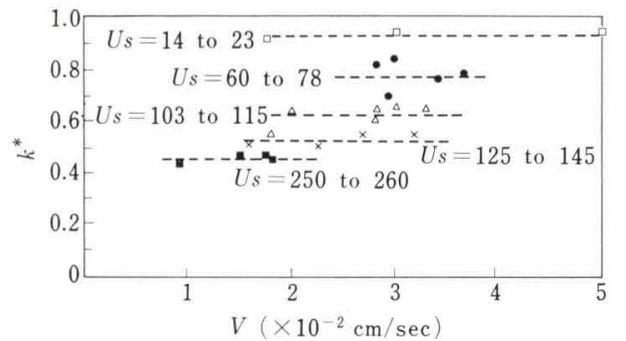

Fig. 3. Relation between effective distribution coefficient, $k^{*}$, and rate of solidification, $V$, for 5 levels of $U s$ in $\mathrm{Al}-4 \mathrm{wt} \% \mathrm{Cu}$ alloy ( $U s$ is peripheral velocity of the rotating solid surface.)

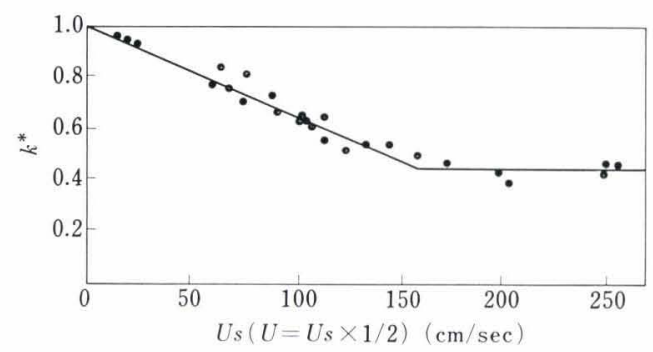

Fig. 4. Relation between the effective distribution coefficient, $k^{*}$, and the peripheral velocity of the rotating solid surface, $U s$, for $\mathrm{Al}-4 \mathrm{wt} \% \mathrm{Cu}$ alloy

For $U<80 \mathrm{~cm} / \mathrm{sec}$

$$
k^{*}=1-6.86 \times 10^{-3} U
$$

For $U \geq 80 \mathrm{~cm} / \mathrm{sec}$

$$
k^{*}=0.45
$$

Thus it is found that Eq. (1), obtained by Burton et al. on the solidification with a planar solid/liquid interface, no longer holds true in the ingot solidification which proceeds with the solidifying zone. The effective distribution coefficient in the solidifying zone will be discussed theoretically in the following section.

\section{Theoretical Consideration on the Solidifying Zone and the Effective Distribution Coefficient}

\section{Classification of the Solidifying Zone}

The solidifying zone can be divided into two zones, $p$ and $q$ according to the previous observation. ${ }^{11)}$ Both the $p$ and $q$ zones are composed of solid and liquid phases. In the $p$ zone the liquid is entrapped by the solid. Thus the concentration of solute in the $p$ zone remains unchanged after solidification. On the other hand, the solid phase exists within the liquid in the $q$ zone. Furthermore, the $q$ zone is subdivided into $q_{1}$ and $q_{2}$ zones. In the $q_{1}$ zone the solid phase forms dendrite skeleton and the liquid can flow through the dendrite arm spacings. The $q_{2}$ zone is the so-called pasty zone containing free dendrites.

The remaining liquid in the $p$ zone becomes the. origin of dendrite segregation and nonmetallic inclusions, since the solute concentration of the remaining liquid is sufficiently increased to form nonmetallic inclusions during solidification. Thus the behaviour of remaining liquid becomes as the most important factor for the discussion concerning the formation mechanism of nonmetallic inclusions. The average solute concentration of the $p$ zone, $\overline{C_{p}}$, must be equal to that of the solid zone, $\overline{C_{s}}$, since it remains constant after solidi-

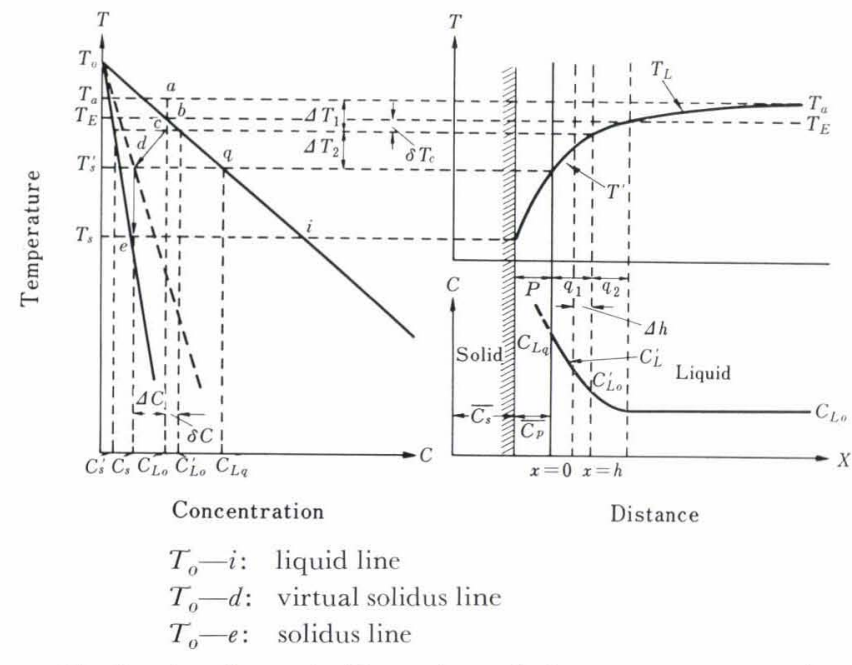

Fig. 5. A schematic illustration of the temperature and the concentration distributions near the solid/liquid interface in binary alloys

fication. Therefore, the $p$ zone can be considered as a virtual solid zone on the discussion of the effective distribution coefficient, $k^{*}$. Only the $q$ zone will be examined in detail later.

\section{Boundary between the $p$ and $q$ Zones}

In order to obtain the boundary condition between the $p$ and $q$ zones let us examine the solidification during which the solidifying zone is completely washed out by the fluid motion of bulk liquid. As the dashed line, $T_{0}-d$, is a virtual solidus line in Fig. 5, it shows the critical fraction of the solid, $S p$, above which the $q$ zone changes to the $p$ zone. The average solute concentration of the $p$ zone, $\overline{C_{p}}$, is expressed as follows:

$$
C_{p}=\delta S k_{o}^{\prime} C_{L o}+(S p-\delta S) k_{o} C_{L o}+(1-S p) C_{L o} \ldots \ldots
$$

where $\delta S$ is the fraction of the solid initially formed when the melt is undercooled by $\delta T_{c}, k_{o}^{\prime}$ the nonequilibrium distribution coefficient, $C_{L o}$ the initial solute concentration of the liquid, and $k_{o}$ the equilibrium distribution coefficient. Since the concentration $C_{p}$ is equal to the concentration $\overline{C_{s}}$, the minimum effective distribution coefficient, $k_{m}^{*}$, is expressed by

$$
k_{m}^{*}=\overline{C_{p}} / C_{L_{o}}=1-S p\left(1-k_{o}\right)+\delta S\left(k_{o}^{\prime}-k_{o}\right)
$$

The critical fraction of solid, $S p$, above which the $q$ zone changes to the $p$ zone, is also represented by,

$$
S p=\frac{\left(1-k_{m}^{*}\right)+\delta S\left(k_{o}^{\prime}-k_{o}\right)}{1-k_{o}}
$$

The term of $\delta S\left(k_{o}^{\prime}-k_{o}\right)$ is supposed to be very small as compared with that of $\left(1-k_{m}^{*}\right)$ in Eq. (8) except in the case of a comparatively large degree of undercooling at the initial stage of solidification. Thus the minimum effective distribution coefficient is approximately given by

$$
k_{m}^{*}=1-S p\left(1-k_{o}\right)
$$

The value of $S p$ is estimated to be over $50 \%$ because it is evident that the solute-rich liquid can flow and 
diffuse at $S p=0.5$ in the previous study for $\mathrm{Al}-\mathrm{Cu}$ alloy ${ }^{11)}$ made by the authors.

\section{Temperature Profile in the $q$ Zone}

In this section, the following five assumptions are made:

(1) The heat conductivity and the specific gravity of the $q$ zone are the mean values between those of the solid and the liquid.

(2) The temperature difference between the solid and the liquid is negligible in the solidifying zone because of their high heat conductivities.

(3) The latent heat of fusion is considered to be the change in the specific heat.

(4) The solidification begins slightly below the equilibrium liquidus temperature.

(5) The fluid flow of molten metal is the so-called "slug flow" since the heat transfer due to the turbulent mixing is negligible on account of the high heat conductivity even when turbulence is caused in the $q_{1}$ zone by the application of stirring. ${ }^{20)}$

Now, the following calculation is made in order to obtain the temperature profile of the zone. If $x$ is the distance along the direction of solidification and the origin is always taken at the point of the virtual solidus line, that is $x=0$ at $T^{\prime}=T_{s}^{\prime}$ in Fig. 5, the equation for steady-state heat flow in the $q$ zone is expressed as follows:

$$
\frac{d^{2} T}{d x^{2}}+\frac{V}{\alpha^{\prime}} \quad \frac{d T^{\prime}}{d x}=0
$$

where $V$ is the rate of solidification, $\alpha^{\prime}$ the thermal diffusivity of the $q$ zone. The boundary conditions are

$$
\begin{array}{ll}
x=0: & T^{\prime}=T_{s}^{\prime} \\
x=h: & T^{\prime}=T_{E}-\delta T_{c}
\end{array}
$$

Assuming that $T^{\prime}$ is finite even at $h=\infty$, the solution of Eq. (10) is given by

$$
T^{\prime}(x)=T_{s}^{\prime}+\underset{1-\exp \left(-V h / \alpha^{\prime}\right)}{J T_{2}}\left\{1-\exp \left(-V x / \alpha^{\prime}\right)\right\}
$$

where

$$
\lrcorner T_{2}=T_{E}-\delta T_{c}-T_{s}^{\prime}
$$

and

$$
\begin{aligned}
\alpha^{\prime} & =\lambda /\left\{\rho \widehat{C_{p}^{-}}+\rho(S p-\delta S) L / \Delta T_{2}\right\} \\
& \approx \lambda \Delta T_{2} / \rho(S p-\delta S) L \ldots \ldots \ldots \ldots
\end{aligned}
$$

Inserting $T_{L}=T_{a}$ at $x=\infty$ and $T_{L}=T_{E}-\delta T_{c}$ at $x=h$ into the equation similar to Eq. (10), the temperature profile of the bulk liquid, $T_{L}(x)$, is expressed as follows:

$$
\begin{array}{r}
T_{L}(x)=T_{a}-\Delta T_{1} \exp V(h-x) / \alpha . \\
\Delta T_{1}=T_{a}-T_{E}+\delta T_{c} \ldots \ldots .
\end{array}
$$

\section{Thickness of the $q_{1}$ Zone}

The starting point of solidification, $x=h$, in Fig. 5 is determined from the boundary condition which can be obtained from Assumption 3 in the previous section

$$
\left(\begin{array}{c}
d T^{\prime} \\
d x
\end{array}\right)_{x=h}-\left(\frac{d T_{L}}{d x}\right)_{x=h}=V \rho \operatorname{LiS} / \lambda
$$

where $\delta S$ is the fraction of the solid initially formed at $x=h$ when the molten alloy is undercooled by $\delta T_{c}$, as described above. On the basis of the equilibrium phase diagram the relation between $\delta S$ and $\delta T_{c}$ is represented by

$$
\delta S=\delta T_{c} /\left(a C_{L o}+\delta T_{c}\right)\left(1-k_{o}\right)
$$

where $C_{L o}$ is the initial solute concentration of the liquid.

From the relation between the degree of undercooling and the solute concentration of the primary crystal in $\mathrm{Al}-4 \mathrm{wt} \% \mathrm{Cu}$ alloy it is obvious that the solute concentration of the primary crystal is nearly consistent with that of the solidus line in the equilibrium phase diagram when the melt is undercooled by less than $15^{\circ} \mathrm{C} .{ }^{21)}$ Thus the fraction of solid of $\delta S$ is calculated from Eq. (18) if the molten alloy is undercooled by less than $15^{\circ} \mathrm{C}$.

Using Eqs. (12), (15), and (17), the thickness of the $q_{1}$ zone is expressed as follows:

$$
h=a_{V}^{\prime} \ln \left(1+\frac{\Delta T_{2} / \alpha^{\prime}}{\rho L \delta S / \lambda+\Delta T_{1} / \alpha}\right)
$$

\section{Fluid Flow in the $q_{1}$ Zone with Stirring}

The fluid flow in the $q_{1}$ zone will only be discussed in this section. It is easy to examine the $q_{2}$ zone on the basis of hydrodynamics, since the $q_{2}$ zone can be treated as a kind of liquid. No experiment on the fluid flow in the $q_{1}$ zone having a complicated structure of dendrites has been made up to present. However, this can be understood to some extent from the experimental results obtained by Nikuradse with coarse sand grains glued on the surface ${ }^{22)}$ as described below.

When all protrusions on the surface of solid are contained within the laminar sub-layer, the rough surface is hydraulically smooth. When protrusions extend partly outside the laminar sub-layer, the transitional turbulence is caused by them. When all protrusions reach outside of the laminar sub-layer, the protrusions experience the friction fully. The height of protrusion, $h$, is only a roughness factor in the above case. However, the distance between protrusions, $b$, must also be taken into consideration as one of the roughness factors. For example, from the experiment on the sphere glued to the surface it was found that the rough surface became hydraulically smooth with the decrease of the distance between protrusions when the distance was very small. ${ }^{23)}$

Accordingly, in the Nikuradse's experiment it is only shown that turbulence can be caused in the $q_{1}$ zone by stirring. The following proposition may be given in the current work, that is, turbulence does not occur in the whole range of $q_{1}$ zone and can not penetrate over a limited distance from the tip of the protrusions, $\Delta h$, when $b / h$ is less than unity. From the photographic observation of the macrostructure in the columnar zone of an $\mathrm{Al}-\mathrm{Cu}$ alloy, it is estimated that 
the value of $b / h$ is considerably smaller than unity. Although $\Delta h$ and the factors controlling it have never been observed or evaluated, it may be considered that $\Delta h$ is proportional to $b$ and is the order of the magnitude of $b$. The value of $\Delta h$ is treated as an experimental constant here and will be examined from the comparison with their experimental results later.

\section{Transport of Solute by Stirring}

The mass (or momentum), which is transported per unit time by turbulent mixing, is expressed as follows:

$$
Q=-A\left(\frac{d W}{d x}\right)
$$

where $W$ is the mass (or momentum) which is transported by turbulent mixing, and $A$ the turbulent diffusivity. The term of $A$ is called as the turbulent diffusivity of mass $\left(A_{D}\right)$ for the transport of mass, and as the turbulent diffusivity of momentum $\left(A_{\tau}\right)$ for the transport of momentum.

The transports of mass and momentum due to diffusion and viscous flow are generally negligible small in the case of turbulent flow. Since $A_{D}$ is approximately equal to $A_{\tau}$ in the turbulent flow, ${ }^{24)}$ the solute concentration of the liquid, $C_{L}^{\prime}$, is proportional to the flow velocity, $U$. The above condition is supposed to hold true in the bulk liquid outside of the $q_{1}$ zone, i.e. $x>h$ in Fig. 5.

The distribution of the solute concentration of the liquid within the $q_{1}$ zone is determined by the temperature profile of the zone. Since the diffusion distance is decreased in the vicinity of $x=0$ and the diffusion by turbulent mixing is violent in the vicinity of $x=h$ in Fig. 5, the solute concentration of the liquid along the direction perpendicular to the $x$ axis is supposed to be almost uniform. So the concentration gradient of solute in the liquid of $q_{1}$ zone will be produced along the $x$ direction. Thus it is approximately expressed as follows:

$$
\frac{d C_{L}^{\prime}}{d x}=-\frac{d T}{d x} / a
$$

The liquid of initial concentration $C_{L O}$ is changed into the solid of $C^{\prime}{ }_{s}$ and the liquid of $C_{L o}^{\prime}$ when the molten alloy is undercooled by $\delta T_{c}$. The ratio of the solid area to the liquid area at the hypothetical interface of $x=h$ in Fig. 5 is considered to be $\delta S /(1-\delta S)$. The solute concentration of the liquid is $C_{L o}^{\prime}$ in the area which is adjacent to the solid surface and $C_{L o}$ in the area which is apart from the solid surface. Thus the average solute concentration of the liquid at $x=h$ is represented by

$$
\left.\begin{array}{rl}
\overline{C_{L o}^{\prime}} & =C_{L o}(1-\delta S)+C_{L o}^{\prime} \delta S \\
& =C_{L o}+\delta C \delta S \approx C_{L o}
\end{array}\right\}
$$

where

$$
\delta C=C_{L o}^{\prime}-C_{L o}
$$

\section{Effective Distribution Coefficient}

Columnar crystals were observed in the macrostructure of $\mathrm{Al}-4 \mathrm{wt}^{\circ} \% \mathrm{Cu}$ alloy in this experiment. So, the effective distribution coefficient in the solidification which is accompanied by the $q_{1}$ zone with columnar protrusions, has been examined in order to analyse the experimental results. When turbulence is caused in the $q_{1}$ zone by stirring, the quantity of solute, which is transported per unit time outside of the $q_{1}$ zone by the turbulent mixing at $x=h$, is expressed from Eqs. (20) and (21) as follows:

$$
(\dot{m})_{x=h}=\frac{A_{D}}{a}\left(j \frac{d T}{d x}\right)_{x=h}
$$

where $j$ is the effective cross section for diffusion and so $(j)_{x=h}=1-\delta S$, as described above. With the advance of solidification, the quantity of solute, which is transported inside of the $q_{1}$ zone, is $V \overline{C_{L o}^{\prime}} \approx V C_{L o}$ at $x=h$, while the quantity of solute, which is transported outside of the $q_{1}$ zone, is $V \overline{C_{p}}=V \overline{C_{s}}$ at $x=0$. Thus Eq. (24) is rewritten as follows:

$$
V\left(C_{L o}-\overline{C_{s}}\right)=(1-\delta S) \frac{A_{D}}{a}\left(\frac{d T^{\prime}}{d x}\right)_{x=h}
$$

Inserting Eq. (12) into Eq. (25), the following equation is obtained.

$$
1-k^{*}=\frac{(1-\delta S) A_{D} \Delta T_{2} \exp \left(-V h / \alpha^{\prime}\right)}{a C_{L o} \alpha^{\prime}\left\{1-\exp \left(-V h / \alpha^{\prime}\right)\right\}}
$$

It has been reported that $A_{\tau}$ is in proportion to the turbulent flow velocity, $U,{ }^{25)}$ and the width of mixing zone, $\Delta h^{26)}$ Since $A_{\tau}=A_{D}$ under the condition of turbulence as described above, $A_{D}$ is given by

$$
\left.A_{D}=B\right\lrcorner h U
$$

where $B$ is a constant determined on the basis of hydrodynamics.

Substituring $\alpha^{\prime}$ of Eq. (14) into Eq. (19), the thickness of the $q_{1}$ zone is represented by

$$
h=\frac{\lambda \Delta T_{2}}{\rho L(S p-\delta S)} \ln (1+Z)
$$

where

$$
Z=\frac{\rho L(S p-\delta S) / \lambda}{\rho L \delta S / \lambda+\Delta T_{1} / \alpha}
$$

Using Eq. (28),

$$
\exp \left(-V h / \alpha^{\prime}\right)=\exp \{-\ln (1+Z)\}=1 /(1+Z) \ldots
$$

Therefore, Eq. (26) is rewritten as follows:

$$
1-k^{*}=\frac{\rho B \Delta h L(1-\delta S)(S p-\delta S) U}{a C_{L_{0}} \lambda Z}
$$

It is confirmed that $\Delta h$ and $\delta S$ remain approximately constant in Eq. (31) except the cases of the initial and later stages of solidification and of the special conditions of slow cooling. Assuming that they are constant, all the factors but $U$ in the righthand side of Eq. (31) become constant. So, the effective distribution coefficient, $k^{*}$, is expressed as follows :

$$
k^{*}=1-E U
$$

where

$$
E=\frac{\rho B \Delta h L(1-\delta S)(S p-\delta S)}{a C_{L o} \lambda Z}
$$


Equation (32) shows that $k^{*}$ decreases linearly with increasing $U$. It will be demonstrated later that there is a lower limit to the value of $k^{*}$ in Eq. (32) because the solidifying zone can not be washed by the fluid motion of bulk liquid when the fraction of solid in the solidifying zone is increased to some extent. The above consideration agrees well with the experimental results of Eqs. (5-a) and (5-b).

\section{Evaluation of the Factors Controlling $\boldsymbol{k}^{*}$}

Equation (32) is the solution for the infinite ingot under the steady state condition. Strictly speaking it is unreasonable to apply this equation for the case of a finite ingot, in which the solidification proceeds under the unsteady state condition. However, Eq. (32) may be adopted to obtain an approximate solution for the finite ingot except the vicinity of the inner and the outer cylinders.

The analytical calculation is compared with the experimental result. Reichardt reported $B=1.4 \times$ $10^{-2}$ for the free turbulence, ${ }^{27)}$ and Karmán also presented $B=1.19 \times 10^{-2}$ for the flow along a smooth wall. ${ }^{28)}$ Both values are approximately the same. The value of $1.2 \times 10^{-2}$ is used here for $B$. The physical constants in Eq. (33) are quoted from the data of aluminum: $\lambda=0.334 \mathrm{cal} / \mathrm{cm} \cdot \mathrm{sec} \cdot{ }^{\circ} \mathrm{C}$, where $\lambda=\left(\lambda_{1}+\lambda_{2}\right)$ $2{ }^{29)} \lambda_{1}=0.247$ for the liquid at $700^{\circ} \mathrm{C}$, and $\lambda_{2}=0.42$ for the solid at $600^{\circ} \mathrm{C},{ }^{30)} \mathrm{c}=0.259 \mathrm{cal} / \mathrm{g} \cdot{ }^{\circ} \mathrm{C}$ at $660^{\circ}$ to $1000^{\circ} \mathrm{C},{ }^{29)} \quad \rho=2.38 \mathrm{~g} / \mathrm{cm}^{3},{ }^{29)}$ and $L=96 \mathrm{cal} / \mathrm{g}^{.29)}$ From the equilibrium phase diagram of $\mathrm{Al}-\mathrm{Cu}$ alloy the following values are obtained: $a=340 / \rho \cdot{ }^{\circ} \mathrm{C} \mathrm{cm}^{3} / \mathrm{g}$, $k_{o}=0.16,{ }^{15)}$ and $\mathrm{C}_{L o}=0.04 \mathrm{~g} / \mathrm{cm}^{3}$.

Then, the factors of $\delta S, S p$, and $\Delta h$ are determined on the basis of the experimental results and the analysis. The fraction of solid, $\delta S$, which is initially formed when it is undercooled by $\delta T_{c}$, is firstly examined. The degree of undercooling, $\delta T_{c}$, remained approximately constant at $4{ }^{\circ} \mathrm{C}$ in a wide region of $\mathrm{Al}-4 \mathrm{wt} \%$ $\mathrm{Cu}$ alloy in this experiment. The value of $\delta S$ is calculated to be 0.27 from Eq. (18).

In order to determine the value of the critical fraction of solid, $S p$, above which the $q$ zone changes to the $p$ zone by Eq. (8), it is necessary to obtain the values of the minimum effective distribution coefficient, $k_{m}^{*}$, and the nonequilibrium distribution coefficient, $k_{0}^{\prime}$. Equation (5-b) shows the value of $k_{m}^{*}$. It is reported that the value of $k_{0}^{\prime}$ for $\mathrm{Al}-4 \mathrm{wt} \% \mathrm{Cu}$ alloy is $0.20 .{ }^{21}$ ) Thus the value of $S p$ is calculated to be 0.67 .

To determine the thickness of mixing zone, $\Delta h$, it is necessary to obtain the values of $E$ and $Z$. Equation (5-a) shows the value of $E$. In this experiment it was also observed that the superheat, $\Delta T_{1}$, at the tapping time diminished when the solidification proceeded to the distance of $1 \mathrm{~cm}$ from the inner cylinder. When $\Delta T_{1}=0$, the value of $Z$ is calculated to be 1.44 from Eq. (29). Thus the value of $\Delta h$ calculated becomes $5.9 \times 10^{-2} \mathrm{~cm}$.

Since the mean interval between the columnar crystals, $b$, was measured to be $8.2 \times 10^{-2} \mathrm{~cm}$ for the stirred ingot of this experiment, $\Delta h$ is approximately equal to $b$ as expected.
3. Application of Experimental Results to the Commercial Steel Ingots

1. Similarity of the Morphology and the Distribution of Dendrites between Steel and Al-Cu Alloy

For the discussion of the solidification mechanism and the segregation behaviour it is necessary to observe experimentally the morphology of solidification interface. The solidification interfaces have been observed for an $\mathrm{Al}-4 \mathrm{wt} \% \mathrm{Cu}$ alloy and a carbon steel ingot, as shown in Photos. 1 and 2 respectively. The solidification interface shown in Photo. 1 (a) was formed as follows. A water-cooled pipe was immersed into the center of the melt. The solidification proceeded from the inner to the outer side. Then the water-cooled pipe with the solidifying zone was pulled up and rotated as soon as possible, to remove the re-

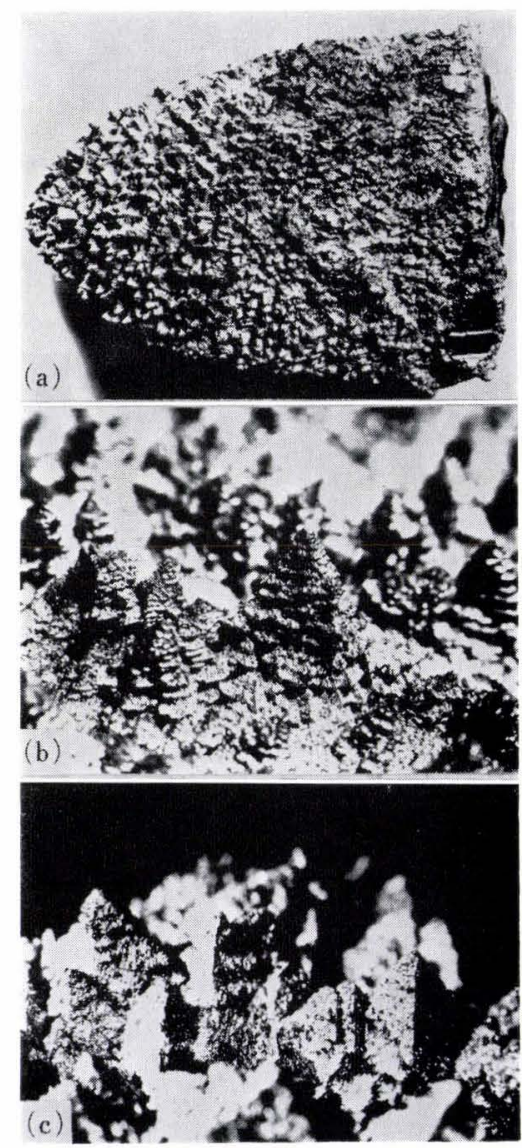

Photo. 1. The morphology of solidification interface for Al$4 \mathrm{wt} \% \mathrm{Cu}$ alloy ingot

(a): $(\times 1)(\times 5 / 8),(b)$ and $(c):(\times 3)(\times 5 / 8)$

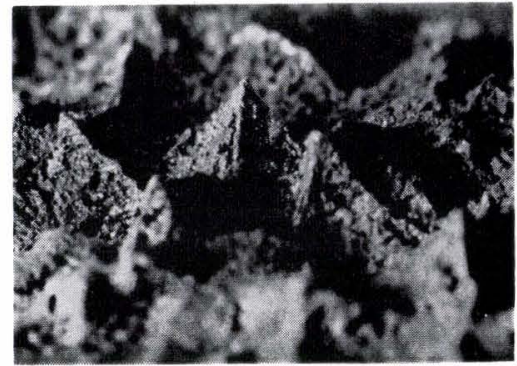

Photo. 2. The morphology of solidification interface for carbon steel ingot $(\times 3)(\times 5 / 8)$ 
maining liquid which had been clinging to the dendritic aggregates. Photographs 1 (b) and (c) show clearly the solidification interface with pyramidal protrusions in larger magnification. Photograph 2 represents the solidification interface with pyramidal protrusions in a steel ingot. It is clearly shown that the solidification interface has the solidifying zone and also the pyramidal protrusions, which are composed of dendritic aggregates in a regular fashion, in steel as well as in $\mathrm{Al}-\mathrm{Cu}$ alloy.

It is also necessary to obtain experimentally the value of the critical fraction of solid, $S p$, above which the liquid is completely entrapped by the solid. Photographic observations were made on the dendrite structure of the vertical sections 3,5 , and $8 \mathrm{~mm}$ apart from the mold in the columnar zone of a $\mathrm{S} 45 \mathrm{C}$ carbon steel ingot of $800 \mathrm{~g}$ in weight and $30 \mathrm{~mm}$ in dia. The distance between the dendrites and the total area of dendrites per unit area were measured in the present investigation. Finally schematic dendrite morphologies were drawn on the basis of the photographic observations. An example of the results obtained is shown in Fig. 6. As the results, $S p$ was decreased from 69 to $65 \%$ with the increase of the distance from the chill. This value agrees well with the value of $S p$ calculated for $\mathrm{Al}-\mathrm{Cu}$ alloy, $67 \%$, as described above. Recently, Tsuda et al. reported that the value of $S p$ is in the range of 53 to $61 \%$ for carbon steels of $120 \mathrm{~kg}{ }^{31}$ )

\section{Effective Distribution Coefficient for Steel}

Mitsuo et al. reported that the flow velocity of free convection is $0.5-10 \mathrm{~cm} / \mathrm{sec}$ by Wojik, $0.17-1.7 \mathrm{~cm} / \mathrm{sec}$ by Mori, and $2.6 \mathrm{~cm} / \mathrm{sec}$ by Romanov, respectively, for the killed steel ingots. ${ }^{32}$ Using Eq. (5-a) and $U$ $=10 \mathrm{~cm} / \mathrm{sec}$ which is the maximum flow velocity of free convection, the value of the effective distribution coefficient is estimated to be more than 0.93 for the killed steel ingot. It is thus evident that the solidifying zone is not washed so much by the free convection of bulk liquid in the case of killed steel ingot. How- ever, it is expected that the effective distribution coefficients of phosphorous and sulphur are increased over unity at the top of the ingot and decreased below

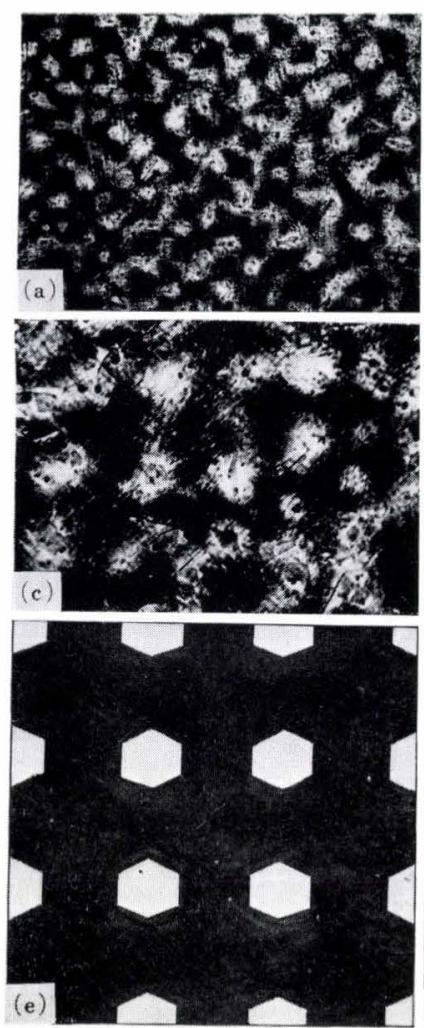

Fraction of solid $68 \%$

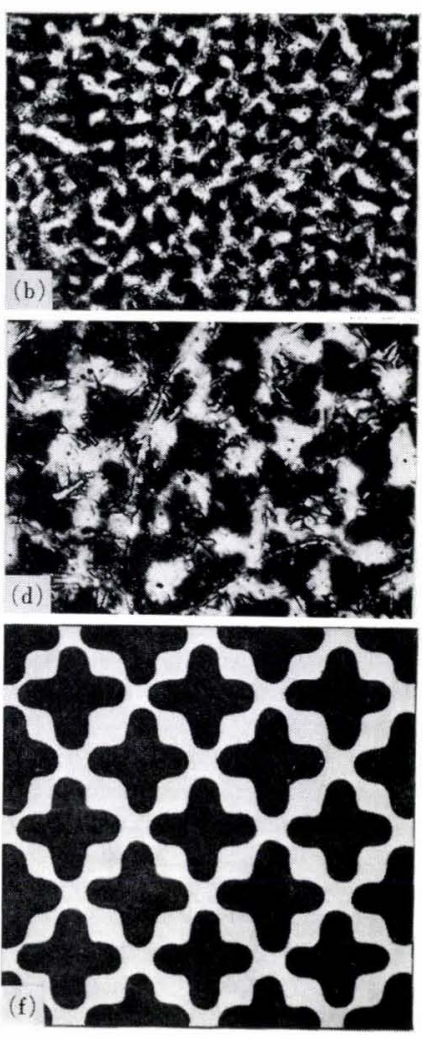

Fraction of solid $63 \%$
Fig. 6. Photographs showing the dendrite structure for carbon steel at the fractions of solid 68 and $63 \%((\mathrm{a})-(\mathrm{d}))$ and schematic illustrations of the dendrite morphology ((e) and (f))

(a) and (b): $(\times 40)(\times 5 / 7)$

(c) and $(\mathrm{d}):(\times 100)(\times 5 / 7)$

Table 1. The minimum effective distribution coefficients in the rim-zone of rimming ingots

\begin{tabular}{|c|c|c|c|c|c|c|c|}
\hline \multirow{2}{*}{$\begin{array}{l}\text { Alloying } \\
\text { element }\end{array}$} & \multicolumn{2}{|c|}{ Equilibrium distribution coefficient } & \multicolumn{5}{|c|}{ Minimum effective distribution coefficient } \\
\hline & $k_{o}$ in $\delta$-crystallization & Ref. & $k^{*}$ (Calculated) & $k^{*}$ & Obser & & Ref. \\
\hline \multirow{4}{*}{ C } & \multirow{4}{*}{0.13} & $(33)$ & \multirow{2}{*}{0.42} & \multirow{2}{*}{\multicolumn{3}{|c|}{$\begin{array}{l}0.42 \\
0.42\end{array}$}} & (39) \\
\hline & & $(34)$ & & & & & $(34)$ \\
\hline & & $(35)$ & 0.46 & \multicolumn{3}{|l|}{0.46} & $(5)$ \\
\hline & & $(6)$ & 0.52 & \multicolumn{3}{|l|}{0.50} & $(40)$ \\
\hline \multirow{4}{*}{ Mn } & 0.72 & $(33)$ & 0.81 & 0.79 & 0.80 & & \multirow{2}{*}{$(41)$} \\
\hline & 0.76 & $(36)$ & 0.84 & 0.85 & 0.90 & 0.91 & \\
\hline & 0.84 & (34) & 0.89 & 0.90 & 0.91 & 0.92 & $(40)$ \\
\hline & 0.90 & $(35)$ & 0.93 & 0.90 & 0.92 & & (34) \\
\hline \multirow{3}{*}{$\mathrm{P}$} & 0.06 & (33) & 0.37 & \multirow{2}{*}{0.38} & \multirow{2}{*}{\multicolumn{2}{|c|}{0.46}} & \multirow{2}{*}{ (41) } \\
\hline & 0.13 & $(34)$ & 0.42 & & & & \\
\hline & $0.2-0.5$ & $(37)$ & $0.46-0.67$ & 0.63 & 0.65 & & $(43)$ \\
\hline \multirow{4}{*}{$\mathrm{S}$} & 0.02 & $(35)$ & 0.34 & 0.33 & 0.34 & 0.37 & (44) \\
\hline & 0.032 & $(6)$ & 0.35 & 0.36 & & \multirow{3}{*}{0.40} & $(45)$ \\
\hline & 0.04 & $(38)$ & 0.36 & 0.38 & 0.39 & & \multirow{2}{*}{ (41) } \\
\hline & 0.05 & $(34)$ & 0.36 & 0.41 & 0.42 & & \\
\hline
\end{tabular}


0.93 at the bottom of the ingot, since a considerable gravity segregation takes place in the solidifying zone. ${ }^{11)}$ It is thus considered that the macrosegregation in the killed steel ingot is controlled partly by the degree of washing against the solidifying zone with the free convection of bulk liquid and partly by the gravity segregation in the solidifying zone.

If the solidifying zone is completely washed out by the fluid motion of bulk liquid during the solidification of alloy, the liquid between dendrites shows the same concentration as the bulk liquid when the fraction of solid is decreased below $67 \%$, i.e. in the $q$ zone. The solute concentration of bulk liquid may increase in the later stage of solidification. But it can be assumed to be the same as the initial solute concentration of the liquid in the early stage of solidification. Then the minimum effective distribution coefficients of carbon, manganese, phosphorous, and sulphur in the steel ingot are calculated by inserting the same critical fraction of solid as obtained for Al-Cu alloy, 67\% and their equilibrium distribution coefficients into Eq. (9). Table 1 shows the calculated and observed values of the minimum effective distribution coefficients. Table 1 clearly shows that these calculated values are approximately equal to the minimum effective distribution coefficients observed for the rim-zone of rimming ingots. It is found that the macrosegregation in the rim-zone of rimmed steel ingot is determined only by the degree of washing against the solidifying zone with the turbulent flow of bulk liquid.

\section{Conclusion}

In order to understand the solidification phenomena of steel ingots, it is most important to clarify the behaviour of the solute-rich liquid which is formed between dendrites in the region of partial solidification, i.e. the solidifying zone. Accordingly, the segregation behaviour and the solidification mechanism were theoretically examined on the basis of a model experiment, in which the morphology of dendritic growth and the fluid flow of bulk liquid during the solidification of $\mathrm{Al}-4 \mathrm{wt} \% \mathrm{Cu}$ alloy were studied. The results obtained are summarized as follows:

The effective distribution coefficient, $k^{*}$, is represented by

$$
k^{*}=1-E U
$$

where $E$ is an experimental constant and $U$ is the flow velocity of bulk liquid. Thus $k^{*}$ depends only on $U$. However, there is a minimum in the value of $k^{*}$. The minimum effective distribution coefficient, $k_{m}^{*}$, is expressed by

$$
k_{m}^{*}=1-S p\left(1-k_{o}\right)
$$

where $S p$ is the critical fraction of solid above which the liquid is completely entrapped by the solid and $k_{0}$ is the equilibrium distribution coefficient. Since the value of $k_{m}^{*}$ is 0.45 for $\mathrm{Al}-4 \mathrm{wt} \% \mathrm{Cu}$ alloy, the value of $S p$ is calculated to be $67 \%$. This agrees well with the value of $S p$ actually observed for carbon steel, 65 to $69 \%$. Assuming that the value of $S p$ is equal to
0.67, the minimum effective distribution coefficients calculated for the alloying elements in steel are obtained as follows: 0.42 for carbon, 0.81 for manganese, 0.37 for phosphorous, and 0.34 for sulphur respectively. These values agree well with the minimum effective distribution coefficients observed for the rim-zone of rimming ingots. From these, it is evident, that the macrosegregation in the rim-zone of rimmed steel ingot is determined only by the degree of washing against the solidifying zone with the turbulent flow of bulk liquid.

\section{List of Symbols}

$a$ : slope of the liquidus line on the phase diagram

$A$ : turbulent diffusivity

$A_{D}: \quad$ turbulent diffusivity of mass

$A_{\tau}$ : $\quad$ turbulent diffusivity of momentum

$b: \quad$ distance between columnar crystals

$B$ : constant determined on the basis of hydrodynamics

$c$ : $\quad$ specific heat of the $q$ zone

$C_{L_{0}}$ : initial solute concentration of the liquid

$\overline{C_{p}}$ : average solute concentration of the $p$ zone

$\overline{C_{s}}: \quad$ average solute concentration of the solid zone

$D$ : diffusion coefficient of the solute

$E$ : empirical constant

$F$ : $\quad$ solidifying zone which is composed of solid and liquid phases

$G$ : temperature gradient

$h$ : thickness of the $q_{1}$ zone

$j$ : $\quad$ effective cross section for diffusion

$k_{o}: \quad$ equilibrium distribution coefficient

$k_{o}^{\prime}$ : nonequilibrium distribution coefficient

$k^{*}: \quad$ effective distribution coefficient

$k_{m}^{*}$ : minimum effective distribution coefficient

$L: \quad$ latent heat of fusion

$(\dot{m})_{x=h}$ : quantity of the solute which is transported per unit time outside of the $q_{1}$ zone by turbulent mixing at $x=h$

$p$ : zone in which the liquid is entrapped by the solid

$q_{1}$ : zone in which the solid forms dendrite skeleton and the liquid can flow through the dendrite arm spacings

$q_{2}$ : $\quad$ zone in which the solid as well as the liquid can flow

Q: $\quad$ mass (or momentum) which is transported per unit time by turbulent mixing

$r$ : distance from the center of the inner cylinder to the fluid

$r_{o}: \quad$ radius of the inner cylinder

Sp: critical fraction of the solid above which the $q$ zone changes to the $p$ zone

$T_{a}$ : tapping temperature

$T_{E}: \quad$ equilibrium liquidus temperature

$T_{s}: \quad$ equilibrium solidus temperature

$T^{\prime}{ }_{s}: \quad$ virtual solidus temperature

$T^{\prime}(x)$ : temperature profile of the $q$ zone

$T_{L}(x)$ : temperature profile of the bulk liquid

$U$ : flow velocity of the bulk liquid 
$U s: \quad$ peripheral velocity of the inner cylinder

$V: \quad$ rate of solidification

$W: \quad$ mass (or momentum) which is transported by turbulent mixing

$x$ : distance from the boundary between the $p$ and the $q_{1}$ zones to the tip of $q_{1}$ zone

$\alpha$ : thermal diffusivity of the bulk liquid

$\alpha^{\prime}: \quad$ thermal diffusivity of the $q$ zone

$\delta$ : thickness of the diffusion layer at the planar interface

$\hat{o}_{l}$ : thickness of the laminar sub-layer

$\delta S$ : fraction of the solid which is initially formed when undercooled by $\delta T$.

$\delta T_{c}: \quad$ degree of undercooling

$\Delta h$ : thickness of the mixing zone in the $q_{1}$ zone

$\rho: \quad$ density of the $q$ zone

$\lambda$ : heat conductivity of the $q$ zone

$\mu$ : viscosity

$\nu$ : kinematic viscosity

$\tau: \quad$ shearing stress

\section{REFERENCES}

1) J. A. Burton, R. C. Prim, and W. P. Slichter: J. Chem. Phys., 21 (1953), 1987.

2) W. A. Tiller, K. A. Jackson, J. W. Rutter, and B. Chalmers: Acta Met., 1 (1953), 428.

3) C. Wagner: Trans. AIME, 200 (1954), 154.

4) W. A. Tiller: Trans. AIME, 224 (1962), 448

5) P. Nilles: JISI, 202 (1964), 601.

6) W. A. Fischer, H. Spitzer, and M. Hishimura: Arch. Eisenhüttenw., 31 (1960), 365.

7) W. A. Fischer and R. Uberoi: Arch. Eisenhüttenw., 33 (1962), 661 .

8) E. Schürmann and W. Grostschel: Arch. Eisenhüttenw., 36 (1965), 619.

9) A. Hultgren and G. Phragmén: Arch. Eisenhüttenw., 12 (1939), 577.

10) H. F. Bishop, F. A. Brandt, and W. S. Pellini: Trans. AFS, 59 (1951), 435.

11) T. Takahashi and I. Hagiwara: J. Jap. Inst. Metals, 29 (1965), 631.

12) I. Hagiwara and T. Takahashi: Tetsu-to-Hagané, 53 (1967), 27.

13) T. Takahashi and I. Hagiwara: J. Jap. Inst. Metals, 29 (1965), 1152

14) T. Takahashi, I. Hagiwara, and K. Ichikawa: Proc. ICSTIS, (1971), 621, Suppl. Trans. ISIJ.

15) M. Hansen: Constitution of Binary Alloys, 2nd ed., (1958), 85, McGraw-Hill, New York.

16) K. Uemura: Tetsu-to-Hagané, 25 (1939), 24.

17) H. Schlichting: Boundary Layer Theory, 4th ed., (1960), 509, McGraw-Hill, New York.
18) G. I. Taylor: Proc. Roy. Soc., A151 (1935), 494.

19) R. R. Miller: Metal Progress, Data Sheet, 59 (1951), No. $1,80-\mathrm{B}$.

20) R. J. Grosh and R. D. Cess: Trans. ASME, 80 (1958), 667.

21) T. Takahashi, T. Suzuki, and K. Ichikawa: J. Jap. Inst. Metals, 32 (1968), 821.

22) J. Nikuradse: V. D. I. Forschungsheft, Nr. 361 (1933).

23) H. Schlichting: Boundary Layer Theory, 4th ed., (1960), 527, McGraw-Hill, New York.

24) E. R. G. Eckert and R. M. Drake, Jr.: Heat and Mass Transfer, (1959), 220; 474, McGraw-Hill, New York.

25) H. Schlichting: Boundary Layer Theory, 4th ed., (1960), 476, McGraw-Hill, New York.

26) H. Schlichting: Boundary Layer Theory, 4th ed., (1960), 481, McGraw-Hill, New York.

27) H. Reichardt: V. D. I. Forschungsheft, 2nd ed,, 414 (1942), 1951.

28) E. R. G. Eckert and R.M. Drake, Jr.: Heat and Mass Transfer, (1959), 226, McGraw-Hill, New York.

29) R. N. Lyon: Liquid Metal Handbook, 2nd ed., (1952), 40, Government Printing Office, Washington D. C.

30) A. G. Guy: Elements of Physical Metallurgy, 2nd ed., (1960), 276, Addison Wesley, Mass.

31) T. Todoroki and K. Tsuda: Report No. 9280-92, Department of Solidification, 19th Committee of Japan Society for the Promotion of Science, May (1971).

32) T. Mitsuo, T. Horigome, S. Saitō, E. Nomura, Y. Kitamura, and R. Kohno: Tetsu-to-Hagané, 55 (1969), S728.

33) K. Guthmann: Stahl u. Eisen, 71 (1951), 399.

34) A. Hays and J. Chipman: Trans. AIME, 135 (1939), 85.

35) C. E. Sims: Electric Furnace Steelmaking, Vol. 2, (1963), 99, John Wiley \& Sons, Inc., New York.

36) T. Wada and H. Wada: Preprint Abstract at the 61st Meeting of Japan Institute of Metals, October (1967), 174 Japan Institute of Metals.

37) R. L. Smith and J. L. Rutherford: J. Metals, 9 (1957), 478.

38) M. Hansen: Constitution of Binary Alloys, 2nd ed., (1958), 85, McGraw-Hill, New York.

39) S. Ishihara: Tetsu-to-Hagané, 40 (1954), 403.

40) I. Kohira: Rimmed Steel Ingots, Reports of Technical Research Institute of Yawata Iron \& Steel Works, Vol. 16, No. 1, Nippon Steel Corp.

41) P. Bardenheuer and C. A. Müller: Mitt. K.-W.-Inst. Eisenforsch., 11 (1929), 225; 273.

42) A. Stadeler and H. J. Tiele: Stahl u. Eisen, 51 (1931), 449.

43) T. Araki and Y. Sugitani: Tetsu-to-Hagané, 56 (1970), 474.

44) M. Imai and J. Matsuno: Report No. 8283-7, Department of Solidification, 19th Committee of Japan Society for the Promotion of Science, December (1966).

45) The Committee of the Iron and Steel Institute, 2nd Report: JISI, 117 (1928), 401. 